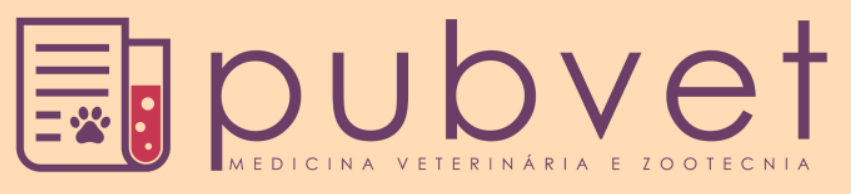

https://doi.org/10.31533/pubvet.v12n9a180.1-9

\title{
El gran impacto de Clostridium perfringens en aves de corral
}

\author{
Daniela Buitrago Mejía ${ }^{1}$, Lina Maria Peñuela $S^{2 *}$, Rosa Angélica Sanmiguel ${ }^{3}$ \\ ${ }^{I}$ Estudiante, Universidad del Tolima, Facultad de Medicina Veterinaria y Zootecnia, Ibagué, Colombia. \\ ${ }^{G}$ Grupo de Investigación en Sistemas Agroforestales Pecuarios, NUTROMICA, Universidad del Tolima, Facultad de Medicina \\ Veterinaria y Zootecnia, Ibagué, Colombia. \\ ${ }^{3}$ Grupo de Investigación IMPRONTA, Universidad Cooperativa de Colombia, Ibagué, Colombia. \\ *Autor para correspondencia. E-mail: Impenuelas@ut.edu.co
}

RESUMEN. Clostridium perfringens tipo A es una bacteria anaerobia Gram positiva que habita en la biota intestinal nativa del intestino grueso de las aves, ocasionando una enteritis necrótica toxémica especialmente en granjas intensificadas de pollos de engorde mayores de 4 semanas de edad, potencialmente zoonótica y que ocasiona altas pérdidas económicas por morbi-mortalidad y disminución de los parámetros productivos. El objetivo de esta revisión fue analizar la problemática generada por la presencia de Clostridium perfringens en sistemas de producción avícola de engorde, a partir de los factores predisponentes del crecimiento bacteriano, patogenicidad y alternativas de tratamiento y control de la enfermedad. Su importancia en el campo avícola nos ha llevado a investigar alternativas para prevenir y controlar la enfermedad producida por Clostridium perfringens en las aves, y así mismo disminuir la proliferación y las pérdidas económicas.

Palabras clave: Enteritis necrótica, enterotoxemia, proliferación

\section{The great impact of Clostridium perfringens in poultry}

ABSTRACT. Clostridium perfringens type A is a Gram-positive anaerobic bacterium that inhabits the intestinal native biota of the intestine of birds. The objective of this review is to analyze different factors that predispose their pathogenicity and bacterial growth, leading to necrotic enteritis, a enterotoxemic disease characterized by hemorrhagic necrosis of the intestinal mucosa, affecting mainly broilers between 4 to 8 weeks of age or older, especially in poultry farms intensified, being a problem for morbidity and mortality hurting performance, weight, food, and due to its presence along the production of broilers, conversion it can be transmitted to humans through the consumption of contaminated poultry products. Its importance in the poultry field has led us to investigate alternatives to prevent and control the disease produced by Clostridium perfringens in poultry, and also reduce the proliferation and economic losses.

Keywords: Enterotoxemic, necrotic enteritis, proliferation

\section{$O$ grande impacto do Clostridium perfringens em aves de capoeira}

RESUMO. Clostridium perfringens tipo A é uma bactéria Gram-positiva anaeróbia que habita a biota intestinal nativa do intestino grosso de aves, causando uma enterotoxemia necrótica, especialmente em sistemas intensivos de frangos de corte com mais de 4 semanas de idade, potencialmente zoonótica e causadora de altas perdas econômicas devido à morbimortalidade e diminuição nos parâmetros de produção. $\mathrm{O}$ objetivo desta revisão foi analisar os problemas gerados pela presença de Clostridium perfringens em sistemas de produção de aves para engorda, com base nos fatores predisponentes de crescimento bacteriano, patogenicidade e tratamentos alternativos e controle da doença. Sua 
importância no campo avícola nos levou a investigar alternativas para prevenir e controlar a doença causada pelo Clostridium perfringens em aves, além de reduzir a proliferação e as perdas econômicas.

Palavras chave: Enterotoxemia, enterite necrótica, proliferação

\section{Introducción}

La enteritis necrótica, o también llamada necrosis intestinal de las aves es una enfermedad generada por la bacteria Clostridium perfringens ocasionando un serio problema para la avicultura por el alto índice de la mortalidad, pudiéndose presentar de forma subclínica en aves que mueren sin mostrar ningún signo; algunos síntomas con los que se presenta son, plumaje erizado, deshidratación, anorexia, disminución del crecimiento, posición en decúbito esternal, aumento de la conversión alimenticia, baja en la producción y finalmente sobreviene la muerte, causando a su vez un impacto significativo en la economía avícola (Craven et al., 2001; García et al., 2014). La enteritis necrótica causa un mal digestivo llamado "apariencia de toalla turca", produce grandes erosiones intestinales incluyendo destrucción severa de la mucosa intestinal generada por un desequilibrio de la microbiota bacteriana del tracto digestivo. La bacteria se encuentra en heces, polvo, agua contaminada, suelo, alimento y camas (Juárez-Estrada, 2014). Debido a su gran importancia y prevalencia en el campo avícola, se realizó una revisión acerca de sus características, su morbilidad, mortalidad, su acción en el intestino del ave, la producción de diferentes toxinas cada una con una diferencia de capacidad de resistencia y patogenicidad y finalmente se describieron posibles métodos para disminuir o controlar la presentación de la enfermedad mediante diferentes opciones.

\section{Generalidades de Clostridium perfringens}

Clostridium perfringens es una bacteria que constituye la principal causa de enteritis necrótica en aves, en especial en las granjas avícolas intensificadas. Esta enfermedad fue reportada por primera vez por Parish (1961) caracterizada por necrosis hemorrágica de la mucosa intestinal. La enterotoxemia necrótica (EN) es una enfermedad que afecta principalmente a pollos de engorde entre 4 a 8 semanas de edad o mayores, aunque también se ha descrito esta enfermedad en pavos, patos y aves silvestres (García et al., 2014; Gomes et al., 2008). Las enfermedades causadas por esta bacteria pueden ser una problemática en las producciones por su nivel de morbilidad y mortalidad y en ocasiones puede actuar como un patógeno oportunista. Clostridium perfringens es una bacteria de interés y cuidado para la industria avícola, ya que es frecuente en aves comerciales con 75 a $95 \%$ del tracto gastrointestinal de pollos de engorde en estudios que han reportado la presencia de Clostridium perfringens y en las aves ponedoras también ha sido demostrado que tienen números relativamente altos de 8 a $84 \%$ de ésta bacteria. Este patógeno entérico, que se encuentra a lo largo de la producción de pollos de engorde, puede ser transmitido a los seres humanos a través del consumo de productos avícolas contaminados (Baba et al., 1997; Craven et al., 2001; McReynolds et al., 2009; Morris \& FernándezMiyakawa, 2009).

Según García et al. (2014) Clostridium perfringens es una bacteria anaeróbica Grampositiva, inmóvil con capacidad de producir esporas y de causar infecciones de origen exógeno y endógeno, se encuentran en diversos sitios de interés, el agua, alimento, ambiente en general o animales homeotermos, siendo parte de la biota intestinal nativa del intestino grueso y sacos ciegos, relativamente inocuos (Barbara et al., 2008) a menos que existan factores predisponentes como la coccidiosis, sometiendo el animal a estrés generando cambios en la flora intestinal, impactación parcial del intestino por ingestión de cama, grano o ambos, cambios en la dieta, restricción alimentaria prolongada, tamaño de la partícula del alimento menos a $4 \mathrm{~mm}$, formulación alimenticia basada en cereales como el trigo, centeno o cebada, dietas con alto contenido de harina de pescado, proteína con altos niveles de energía y uso de proteínas de baja digestibilidad favoreciendo el crecimiento de Clostridium perfringens.

\section{Patogenia}

Clostridium perfringens es un microorganismo que presenta alto grado de intercambio genético; esto le permite la transferencia de factores de virulencia y le otorga la capacidad de producir las diferentes toxinas como resultado de la pérdida o la ganancia de los genes específicos. En definitiva, la universalidad de Clostridium perfringens sumada a la independencia de los factores de virulencia denota el potencial patogénico de esta bacteria, especialmente si se tiene en cuenta que es 
parte de la microbiota del intestino de las aves (Canard \& Cole, 1989; Morris \& FernándezMiyakawa, 2009).

La virulencia de Clostridium perfringens se debe a la producción de toxinas alfa (Clostridium perfringens tipo A), Beta (tipo B), épsilon (tipo C) $\mathrm{y}$ iota (tipo E) y también de un repertorio compuesto, hasta el momento, de 15 toxinas proteicas. Dentro del resto de las toxinas no utilizadas en la clasificación, pero importantes desde el punto de vista patológico, se encuentra la enterotoxina de Clostridium perfringens o CPE, responsable de diarreas en humanos y animales; siendo este síntoma predominante de Clostridium perfringens, la recientemente descubierta toxina NetB, relacionada con la enteritis necrótica en aves y la toxina beta-2, aparentemente asociada a ciertos cuadros de enteritis (Keyburn et al., 2008). Clostridium perfringens tipo A, toxina alfa es el principal factor de virulencia de la gangrena gaseosa en humanos, animales y de la enteritis necrótica en pollos como en otros animales domésticos. La toxina tipo B es responsable de algunas enfermedades de origen intestinal como ulceraciones en el intestino delgado y la toxina tipo $\mathrm{C}$ causa enteritis necrótica. En los animales infectados se observa diarrea y disentería, con materia fecal sanguinolenta y muerte. Esta bacteria también produce enterocolitis necrótica en humanos, una enfermedad potencialmente letal (Johnson \& Gerding, 1997). En el tipo E Los animales afectados presentaban enteritis necrótica y hemorrágica aguda (Morris \& FernándezMiyakawa, 2009).

El descubrimiento de una toxina formadora de poros (NetB), presente en cepas de Clostridium perfringens tipo A aisladas de aves con enteritis necrótica, abre una nueva línea de investigación, relevante tanto para el conocimiento de la patogenia de estos microorganismos como para el desarrollo de nuevas vacunas (Keyburn et al., 2008). Esta toxina, o tal vez otras también, podrían estar involucradas y juegan un papel importante en la enteritis necrótica por Clostridium perfringens tipo A observada en otras especies animales (Morris \& Fernández-Miyakawa, 2009). En el caso del papel exacto de la toxina beta-2 codificado por un gen (cpb2) producida por Clostridium perfringens en la patogénesis de EN sigue siendo controvertido (Tolooe et al., 2011). En el caso de la producción avícola, Sherman et al. (1994) reporta que la cepa más importante es el tipo A, ya que es responsable de la mayoría de los procesos infecciosos. Produce una enterotoxina que se comporta como un súper antígeno promoviendo la liberación de mediadores de inflamación en forma masiva. La enterotóxina tiene actividad letal, citotóxica y enterotóxica. En el intestino delgado, produce daño morfológico y fisiológico, lo cual originaría la diarrea. El mecanismo de muerte celular es dependiente de la concentración de toxina y de la concentración de ciertos iones extracelulares, como el calcio. La evidencia existente indica que la apoptosis sería el mecanismo predominante a bajas concentraciones y la necrosis a altas concentraciones. En ambos casos la enterotoxina produce daño histopatológico en el intestino, lo que lleva a la pérdida de electrolitos y fluidos que se observa en modelos experimentales. Según Annett et al. (2002) EN por Clostridium perfringens tipo A es un problema persistente que afecta de 1.3 a $37.3 \%$ el rápido crecimiento en pollos, generando pérdidas económicas directas e indirectas. Varios factores se saben para precipitar brotes de NE, incluyendo altos niveles dietéticos de trigo, cebada o la harina de pescado y daños a la mucosa intestinal debido a desperdicios de alto contenido de fibra o coccidiosis. La necrosis intestinal característica del NE es causada por la potente toxina $\alpha$ producida por $C$. perfringens.

\section{Relación de algunas enfermedades con enteritis necrótica}

\section{Inmunosupresión}

La exposición a la enfermedad infecciosa de la bolsa de Fabricio, el virus de la anemia infecciosa y la enfermedad de Marek, así como el estrés no específico, puede predisponer a las aves a EN (Schuring \& van Gils, 2001). La enteritis necrótica se ha correlacionado con la presencia de otras enfermedades infecciosas, tal como coccidiosis y enfermedad de la bursa (Islam et al., 2009; McReynolds et al., 2004).

La coccidiosis causa inmunosupresión y daño gastrointestinal severo, que dan lugar a Clostridium perfringens, ofreciendo los sustratos de nutrientes ricos en proteínas favorables a Clostridium perfringens proliferación y producción de toxinas. Long (1973) informó que 75\% de las aves diagnosticadas con EN también estaban infectadas con coccidias, principalmente por Eimeria brunetti, Eimeria maxima o Eimeria tenella y Eimeira necatrix (Baba et al., 1997) y Eimeira Acervulina, que genera una alta mortalidad, hasta un $53 \%$ en aves infectadas, según (Al-Sheikhly \& Al-Saieg, 1980) mostrando 
que Clostridium Perfringes genera diferentes grados de mortalidad dependiendo de la cepa de Eimeria que esté presentes en el ave. La combinación de la coccidiosis y la enteritis necrótica podría tener efectos devastadores en morbilidad y mortalidad en la industria avícola comercial, permitiendo que Clostridium perfringens se replique rápidamente y produzca la toxina, probablemente por la fuga de proteínas incluyendo las del plasma en el lumen del intestino durante la infección de Eimeria (Bahram et al., 2012).

Por otro lado, la bursitis infecciosa en las aves de corral, es un virus que afecta a las células linfoides y, específicamente, se dirige a la bolsa de Fabricio, que es responsable para el desarrollo de la inmunidad humoral en pollos. Los polluelos infectados con esta enfermedad a menudo tienen infecciones secundarias tales como Clostridium perfringens exacerbando la enfermedad (McReynolds et al., 2004). EN según McReynolds et al. (2004) puede dividirse en 2 categorías: clínica y subclínica. En los signos clínicos incluyen una característica destacada de la enteritis necrótica que es aguda muerte, con tasas de mortalidad que puede alcanzar el 50\% (van Immerseel et al., 2004), depresión, disminución del apetito, diarrea y la necrosis severa del tracto intestinal, a la necropsia la mucosa intestinal de estas aves presenta la apariencia de una "toalla turca" (Zavala, 2007). En una encuesta realizada en el año 2000, se estimó que la forma subclínica de la enfermedad cuesta al productor tanto como 5 centavos por ave, principalmente porque la enfermedad clostridiana subclínica puede ser mucho más difícil de identificar y con frecuencia se ignora porque no se reconoce, se puede confundir con otros problemas de salud o bien coexistir con ellos (Zavala, 2007). En esta forma subclínica se puede presentar disminución en la ganancia de peso y empeorar la conversión alimenticia, a causa de disminución de la digestión y absorción, debido al daño del intestino. En combinación con la estimación de producción de carne para asar de 1999, el costo de esta enfermedad, incluyendo infecciones clínicas y subclínicas, fue de cerca de 2 billones de dólares en todo el mundo (Collier et al., 2003; SchockenIturrino et al., 2013).

Entender la progresión de la enfermedad de EN ha sido muy difícil debido a su complejidad y porque varios factores predisponentes tales como componentes de la dieta, inmunosupresión, irritación y cambios de la microflora intestinal súbita parecen contribuir a este síndrome.

Se ha demostrado que componentes de base y cambios bruscos de ración pueden alterar la población microbiana nativa y permitir la colonización por bacterias oportunistas como Clostridium perfringens (Branton et al., 1997; McReynolds et al., 2004). Generalmente la severidad de las lesiones EN están estrechamente relacionadas con la ganancia de peso y la mortalidad (Lee et al., 2011). La enfermedad debida a la producción de la enterotoxina (CPE) (Sarker et al., 1999), se produce en el intestino después de la ingestión de al menos $10^{7}$ células de Clostridium perfringens. Cerca de 8-12 h (6-24 h) después de ingerir alimentos contaminados, los síntomas comienzan con dolor abdominal agudo, náuseas y diarrea. Los alimentos contaminados son casi siempre tratados térmicamente, matando la flora competidora mientras que las esporas de Clostridium perfringens sobreviven. $\mathrm{La}$ enfermedad es auto limitada y tiene una duración de $24 \mathrm{~h}$ aproximadamente. Las muertes pueden producirse debido a deshidratación, vista principalmente en aves adultas o muy jóvenes (Brynestad \& Granum, 2002). EN por alimentos suministrados: Clostridium perfringens puede ser tomado desde el medio ambiente por medio de fuentes que incluyen: alimentos contaminados, agua o cualquier parte de la planta de producción de pollos (Craven et al., 2001; van Immerseel et al., 2004).

El uso de la dieta de cereales granos con alto contenido de polisacáridos sin almidón (NSP), como el trigo, la cebada, el centeno y avena, pueden ser un factor importante, dando lugar a la colonización de Clostridium perfringens en aves (Collier et al., 2003). La enteritis necrótica causada por Clostridium perfringens tipo A, es más frecuente en los pollos alimentados con dietas a base de trigo o cebada, que en los alimentos que contienen maíz (Annett et al., 2002) y es debido a la diferencia en la proliferación de los clostridios en las dietas, lo que resulta en un mayor número de bacterias en el intestino y la falta de fluidez y digestión del trigo y cebada, generando un aumento de la incidencia de la enteritis necrótica en pollos alimentados con dietas de cebada y trigo mediante el aumento de la viscosidad de la digesta, producción de moco y bacterias en crecimiento. Un estudio realizado por Branton et al. (1997) verificó que las lesiones de enteritis necrótica en pollos fueron más bajas en los animales que consumieron la dieta de maíz sin Clostridium 
perfringens y el más alto en los animales alimentados con las dietas de trigo con Clostridium perfringens. Por otro lado, las proteínas animales, como la harina de pescado especialmente, mejoran la proliferación y la producción de toxinas. (Bahram et al., 2012)

\section{Textura física y forma de alimentación}

Según Yegani and Korver (2008) la forma física de los componentes del cereal de la alimentación puede afectar las características morfológicas y fisiológicas del tracto intestinal (Engberg et al., 2012). Finalmente, el alimento molido fino puede aumentar la mortalidad asociada a enteritis necrótica en comparación con el alimento molido grueso. Branton et al. (1997) observó que el uso de trigo molido con un molino de martillos (finamente molido) aumentó la mortalidad a $28,9 \%$, pero una dieta de trigo con molino de rodillos (molido grueso) resultó en una mortalidad de $18,1 \%$. Mortalidad que fue asociada con una combinación de EN y coccidiosis. Algunos estudios muestran que la dieta de trigo integral puede contribuir al rendimiento en pollos, a través del desarrollo del tracto GI, especialmente la molleja, y también mayor absorción de nutrientes en la dieta por el tracto digestivo inferior (Engberg et al., 2012). La alimentación de trigo integral para pollos de engorde reduce el número de Clostridium perfringens en el tracto intestinal de las aves (Engberg et al., 2012; Yegani \& Korver, 2008). La falta de higiene general, la manipulación y el almacenamiento de los alimentos son asociados a la presentación de infección por Clostridium perfringens en los sistemas de producción avícola (SchockenIturrino et al., 2010) por lo que se hace necesario realizar seguimiento periódico de la presencia de Clostridium perfringens en estas fuentes. Desde este punto de vista, el uso de peróxido de hidrógeno en el agua de suministrada a pollos de engorde es una alternativa interesante ya que elimina los microorganismos, mientras que el uso del cloro solamente reduce la cantidad de Clostridium perfringens (Matiz \& Cruz, 2007).

\section{Medidas de control para EN}

Después de la aparición de un brote, el fosfato de tilosina es una alternativa viable para la reducción de la morbilidad, mortalidad y supresión de la eficiencia del crecimiento asociado con EN en pollos de engorde. Administrar 100 ppm de fosfato de tilosina en el pienso reduce significativamente la mortalidad y en las aves sobrevivientes reduce las lesiones (Brennan et al., 2001). EN, especialmente en pollos de engorde, ha sido durante mucho tiempo controlado por algunos antibióticos promotores del crecimiento (AGPs) en el alimento, que incluyen fármacos antibacterianos conocidos como virginiamicina, bacitracina, y así sucesivamente. Sin embargo, normas en la Unión Europea (UE) se opone al uso de ciertas AGPs y el resto han sido prohibidas ( $\underline{\mathrm{van}}$ Immerseel et al., 2004). Algunos anticoccidiales ionóforo en la alimentación, que adicionalmente poseen actividad antibacteriana Gram -positivas, tienen también contribución en enteritis necrótica para el control de pollos de engorde, pero por lo general, se omiten de las dietas de aves de corral, en tal caso, se administra la vacuna anticoccidial (Williams, 2005).

La retirada voluntaria o legal de la utilización de determinados factores de crecimiento, antibióticos en el pienso con la actividad anticlostridial, ha permitido la búsqueda de otras alternativas para controlar o prevenir la EN producida por Clostridium perfringens. El uso de anticoccidiales ionóforos en la alimentación posee actividad anticlostridial, siendo sugeridos para la prevención de EN por Clostridium perfringens, sin embargo, Olazábal et al., (2005) mencionan que el tratamiento con amprolium no es efectivo para esta función. Los anticoccidiales ionóforos, aunque no prohibidos, se impiden por lo general cuando se utilizan vacunas anticoccidiales en vivo, aumentando potencialmente el riesgo de EN (Williams, 2005). Otro tratamiento eficaz que han estudiado ha sido el uso de BMD (bacitracina metileno Disalicilato) y narasina, alimentado solo y en combinación, siendo eficaces en reducir la morbilidad, la mortalidad y la supresión del crecimiento y la eficiencia de alimentación asociado con NE entre pollos de engorde desafiados con Clostridium perfringens (Brennan et al., 2003). Los brotes de NE se pueden prevenir o tratar mediante el uso de antibióticos en piensos, la tilosina puede controlar NE a través de su modulación de la colonización de Clostridium perfringens y la actividad mucolítica de la microbiota intestinal, pero muchos de los antibióticos promotores del crecimiento en los piensos y los anticoccidiales ionóforos han sido retirados, o prohibidos por muchos países, generando una mayor incidencia de NE y dando lugar a la enfermedad entérica bacteriana clínicamente más dramática de las aves de corral, siendo una gran preocupación para los productores de aves de corral (Cooper et al., 2009). Esta re- 
emergencia ha llevado a una búsqueda de métodos alternativos para el control de la enfermedad, en particular la vacunación, para reducir la necesidad actual y además prevenir o tratar la enfermedad en pollos de engorde con fármacos antimicrobianos, ofreciendo un enfoque alternativo para el control de la enfermedad, aunque por el momento se conoce poco sobre la inmunidad de la NE (Collier et al., 2003; Kulkarni et al., 2007).

Otras estrategias para controlar la NE en ausencia de antibióticos promotores del crecimiento, sin tener que recurrir al uso de tratamiento profiláctico o terapéutico, corresponden a prácticas dietéticas y de gestión (Dahiya et al., 2006). Entre los reemplazos candidatos para los antibióticos son productos competitivos de exclusión como lo son los probióticos y prebióticos, donde Abudabos et al. (2015) indican que el uso de éstos, son alternativas a promotores de crecimiento antimicrobianos (AGPs), mejorando el rendimiento de pollos de engorde que puede explicarse en parte por la mejora en la morfología intestinal y el equilibrio microbiano asociado con la modulación de la microflora intestinal y la inhibición de patógenos.

Los ácidos orgánicos, enzimas, extractos de plantas, anticuerpos de huevo de gallina, bacteriófagos y la vacunación ya antes mencionada, la composición química de la dieta en relación con la selección de granos de cereales, fuente de proteína y perfil de aminoácidos puede influir en la propensión de la enfermedad, esto podría ser útil en la formulación de las dietas de aves de corral que reducen la incidencia de EN. Entre las vacunas que se están evaluando, Cooper et al. (2009) determinó en caso tal el uso de la vacunación de pollos de engorde con la toxina alfa recombinante que según sugiere puede servir como un inmunógeno eficaz, y, como tal, puede jugar un papel en la patogénesis. Un enfoque disponible recientemente en los Estados Unidos es la vacuna conocida oficialmente como toxoide Clostridium perfringens tipo A, administrada a las reproductoras pesadas para proteger inmunológicamente a su progenie contra Clostridium perfringens. Como la primera en su tipo en la industria avícola, esta vacuna contra la enteritis necrótica fue desarrolla por ScheringPlough Animal Health y actualmente se está utilizando bajo una licencia condicional en Estados Unidos (Zavala, 2007). También existe una vacuna atenuada de Salmonella que expresa antígenos de Clostridium perfringens e inmuniza pollos de engorde contra la EN vía oral (Kulkarni et al., 2008).

Thompson et al. (2006) utilizaron cepas atenuadas de Clostridium perfringens que ya no expresan una toxina para vacunar pollos. Ellos encontraron que las cepas de vacunas atenuadas ofrecen diferentes niveles de protección. Este estudio claramente demuestra que Clostridium perfringens lleva otras moléculas que pueden proporcionar cierta eficacia como antígenos de vacunas. Los trabajos posteriores de este grupo identificaron varias proteínas immunoreactivas en cepas de Clostridium perfringens virulento que, en forma de subunidad recombinante, ofrecen cierta protección cuando se utilizan como vacunas (Kulkarni et al., 2007). Dos de estas proteínas, aldolada de fructosabifosfato y una proteína hipotética (posiblemente una metaloproteasa), dio cierta protección contra NE al ser entregada por un vector Salmonella vivo (Kulkarni et al., 2008). Otras proteínas que demostraron tener un papel en patogenesia y vale la pena probarlos como antígenos vacunales, incluyeron la proteína de la NetB identificada por Keyburn et al. (2008) y las metaloproteinasas que se sugieren para tener un papel clave en el desarrollo temprano de la lesión (Olkowski et al., 2008; van Immerseel et al., 2009).

El uso de cal virgen para el control de Clostridium spp en camas de aviario según (DaiPra et al., 2009) permite una reducción hasta del $97 \%$ en dosis $300 \mathrm{~g} \mathrm{~m}^{-2}$ y una reducción del $100 \%$ en dosis de 600 e $900 \mathrm{~g} \mathrm{~m}^{-2}$, debido a que la cal eleva el $\mathrm{pH}$, creando un entorno inhóspito para las bacterias, además de actuar mediante la reducción de la cantidad de agua libre y la reducción de la actividad de agua en la camada.

Un estudio realizado por Sadeghi et al. (2015) menciona un método inteligente para la detección de pollos infectados Clostridium perfringens tipo A en base a su vocalización, esto permitió identificar que la vocalización de pollos sanos presentó mayor intensidad y uniformidad en la forma de vocalización que los no saludables, demostrando que la vocalización de los pollos infectados presenta menor intensidad de sonido que las sanas. Esto demuestra la utilidad y la eficacia de los métodos inteligentes para el diagnóstico de enfermedades de aves de corral, permitiendo aplicar un tratamiento temprano a las aves enfermas antes que la enfermedad se difunda más. 


\section{Referencias bibliográficas}

Abudabos, A. M., Al-Batshan, H. A., \& Murshed, M. A. 2015. Effects of prebiotics and probiotics on the performance and bacterial colonization of broiler chickens. South African Journal of Animal Science, 45(4), 419-428.

Al-Sheikhly, F., \& Al-Saieg, A. 1980. Role of coccidia in the occurrence of necrotic enteritis of chickens. Avian Diseases, 24(2), 324-333.

Annett, C. B., Viste, J. R., Chirino-Trejo, M., Classen, H. L., Middleton, D. M., \& Simko, E. 2002. Necrotic enteritis: effect of barley, wheat and corn diets on proliferation of Clostridium perfringens type A. Avian Pathology, 31(6), 598-601.

Baba, E., Ikemoto, T., Fukata, T., Sasai, K., Arakawa, A., \& McDougald, L. R. 1997. Clostridial population and the intestinal lesions in chickens infected with Clostridium perfringens and Eimeria necatrix. Veterinary Microbiology, 54(3-4), 301-308.

Bahram, S., Vince, A. R., \& Prescott, J. F. 2012. The successful experimental induction of necrotic enteritis in chickens by Clostridium perfringens: a critical review. Veterinary Research, 43(1), 74.

Barbara, A. J., Trinh, H. T., Glock, R. D., \& Songer, J. G. 2008. Necrotic enteritisproducing strains of Clostridium perfringens displace non-necrotic enteritis strains from the gut of chicks. Veterinary Microbiology, 126(4), 377-382.

Branton, S. L., Lott, B. D., Deaton, J. W., Maslin, W. R., Austin, F. W., Pote, L. M., . . Day, E. J. 1997. The effect of added complex carbohydrates or added dietary fiber on necrotic enteritis lesions in broiler chickens. Poultry Science, 76(1), 24-28.

Brennan, J., Moore, G., Poe, S. E., Zimmermann, A., Vessie, G., Barnum, D. A., \& Wilson, J. 2001. Efficacy of in-feed tylosin phosphate for the treatment of necrotic enteritis in broiler chickens. Poultry Science, 80(10), 1451-1454.

Brennan, J., Skinner, J., Barnum, D. A., \& Wilson, J. 2003. The efficacy of bacitracin methylene disalicylate when fed in combination with narasin in the management of necrotic enteritis in broiler chickens. Poultry Science, 82(3), 360-363.

Brynestad, S., \& Granum, P. E. 2002. Clostridium perfringens and foodborne infections.
International Journal of Food Microbiology 74, $195-202$.

Canard, B., \& Cole, S. T. 1989. Genome organization of the anaerobic pathogen Clostridium perfringens. Proceedings of the National Academy of Sciences, 86(17), 66766680.

Collier, C. T., van der Klis, J. D., Deplancke, B., Anderson, D. B., \& Gaskins, H. R. 2003. Effects of tylosin on bacterial mucolysis, Clostridium perfringens colonization, and intestinal barrier function in a chick model of necrotic enteritis. Antimicrobial Agents and Chemotherapy, 47(10), 3311-3317.

Cooper, K. K., Trinh, H. T., \& Songer, J. G. 2009. Immunization with recombinant alpha toxin partially protects broiler chicks against experimental challenge with Clostridium perfringens. Veterinary Microbiology, 133(12), 92-97.

Craven, S. E., Stern, N. J., Bailey, J. S., \& Cox, N. A. 2001. Incidence of Clostridium perfringens in broiler chickens and their environment during production and processing. Avian Diseases, 45(4), 887-896.

Dahiya, J. P., Wilkie, D. C., van Kessel, A. G., \& Drew, M. D. 2006. Potential strategies for controlling necrotic enteritis in broiler chickens in post-antibiotic era. Animal Feed Science and Technology, 129(1-2), 60-88.

Dai-Pra, M. A., Corrêa, É. K. n., Xavier, E. G., Lopes, D. C. N., Lourenço, F. F., Zanusso, J. T., \& Roll, A. P. 2009. Uso de cal virgem para o controle de Salmonella spp. e Clostridium spp. em camas de aviário. Ciência Rural, 39(4), 1189-1194.

Engberg, R. M., Grevsen, K., Ivarsen, E., Fretté, X., Christensen, L. P., Højberg, O., . . . Canibe, N. 2012. The effect of Artemisia annua on broiler performance, on intestinal microbiota and on the course of a Clostridium perfringens infection applying a necrotic enteritis disease model. Avian Pathology, 41(4), 369-376.

García, H. G., Ledesma, M. N., Godoy, S. F., \& Urquiza, B. O. 2014. Determinación de la cantidad de Clostridium oerfringens y la Longitud de las vellosidades intestinales en pollos de engorda de 6 semanas de edad aparentemente sanos. Los Avicultores y su Entorno, 100, 136-142.

Gomes, A. M., Lobato, F. C. F., Martins, N. R. S., \& Assis, R. A. 2008. Genotipicação de Clostridium perfringens isolados de frangos de 
corte através da PCR múltipla. Ciência Rural, 38(7), 1943-1947.

Islam, M. N., Rashid, S. M. H., Juli, M. S. B., Hoque, M. F., \& Akhter, M. R. 2009. Necrotic enteritis in chickens: Pathological, bacteriological and therapeutical investigation. International Journal of Sustainable Crop Production, 4(3), 1-8.

Johnson, S., \& Gerding, D. N. 1997. Enterotoxemic infections. In J. I. Rood, J. G. Songer, B. A. McClane \& R. W. Titball (Eds.), The Clostridia - Molecular Biology and Pathogenesis (Vol. 1, pp. 117-140). USA: Elsevier.

Juárez-Estrada, M. A. 2014. La importancia de la Enteritis Necrótica en la salud de las aves actuales. Los avicultores y su entorno No. 74.

Keyburn, A. L., Boyce, J. D., Vaz, P., Bannam, T. L., Ford, M. E., Parker, D., . . Moore, R. J. 2008. NetB, a new toxin that is associated with avian necrotic enteritis caused by Clostridium perfringens. PLoS Pathogens, 4(2), e26.

Kulkarni, R. R., Parreira, V. R., Sharif, S., \& Prescott, J. F. 2007. Immunization of broiler chickens against Clostridium perfringensinduced necrotic enteritis. Clinical and Vaccine Immunology, 14(9), 1070-1077.

Kulkarni, R. R., Parreira, V. R., Sharif, S., \& Prescott, J. F. 2008. Oral immunization of broiler chickens against necrotic enteritis with an attenuated Salmonella vaccine vector expressing Clostridium perfringens antigens. Vaccine, 26(33), 4194-4203.

Lee, K. W., Lillehoj, H. S., Jeong, W., Jeoung, H. Y., \& An, D. J. 2011. Avian necrotic enteritis: experimental models, host immunity, pathogenesis, risk factors, and vaccine development. Poultry Science, 90(7), 13811390.

Long, J. R. 1973. Necrotic enteritis in broiler chickens. I. A review of the literature and the prevalence of the disease in Ontario. Canadian Journal of Comparative Medicine, 37 , 302 308.

Matiz, D. M., \& Cruz, J. L. G. 2007. Evaluación de la calidad del agua (microbiológica y fisicoquímica) en pollos de engorde con el uso del peróxido y cloro. Plumazos, 35, 4-10.

McReynolds, J., Waneck, C., Byrd, J. A., Genovese, K., Duke, S., \& Nisbet, D. 2009. Efficacy of multistrain direct-fed microbial and phytogenetic products in reducing necrotic enteritis in commercial broilers. Poultry Science, 88(10), 2075-2080.

McReynolds, J. L., Byrd, J. A., Anderson, R. C., Moore, R. W., Edrington, T. S., Genovese, K. J., . . . Nisbet, D. J. 2004. Evaluation of immunosuppressants and dietary mechanisms in an experimental disease model for necrotic enteritis. Poultry Science, 83(12), 1948-1952.

Morris, W. E., \& Fernández-Miyakawa, M. E. 2009. Toxinas de Clostridium perfringens. Revista Argentina de Microbiología, 41(4), 251-260.

Olkowski, A. A., Wojnarowicz, C., Chirino-Trejo, M., Laarveld, B., \& Sawicki, G. 2008. Subclinical necrotic enteritis in broiler chickens: novel etiological consideration based on ultrastructural and molecular changes in the intestinal tissue. Research in Veterinary Science, 85(3), 543-553.

Parish, W. E. 1961. Necrotic enteritis in the fowl. I. Histopathology of the examination of the causal Clostridium perfringens II.The experimental diseases. Journal of Comparative Pathology 71, 377-404.

Sadeghi, M., Banakar, A., Khazaee, M., \& Soleimani, M. 2015. An intelligent procedure for the detection and classification of chickens infected by clostridium perfringens based on their vocalization. Revista Brasileira de Ciência Avícola, 17(4), 537-544.

Sarker, M. R., Carman, R. J., \& McClane, B. A. 1999. Inactivation of the gene (cpe) encoding Clostridium perfringens enterotoxin eliminates the ability of two cpe-positive $C$. perfringens type A human gastrointestinal disease isolates to affect rabbit ileal loops. Molecular Microbiology, 33(5), 946-958.

Schocken-Iturrino, R. P., Vittori, J., BeraldoMassoli, M. C., Delphino, T. P. C., \& Damasceno, P. R. 2010. Clostridium perfringens em rações e águas fornecidos a frangos de corte em granjas avícolas do interior paulista: Brasil. Ciência Rural, 40(1), 197-199.

Schocken-Iturrino, R. P., Vittori, J., Massoli, M. C. B., \& Gama, L. F. S. A. M. 2013. Presença de Clostridium perfringens em frangos de corte provenientes de aviários da região de Ribeirão Preto-SP. Ars Veterinaria, 29(1), 37-41.

Schuring, M., \& van Gils, B. 2001. Clostridium perfringens - a nutritional challenge? Feed Mix, 9(1), 26-28. 
Sherman, S., Klein, E., \& McClane, B. A. 1994. Clostridium perfringens type A enterotoxin induces tissue damage and fluid accumulation in rabbit ileum. Journal of Diarrhoeal Diseases Research, 12(3), 200-207.

Thompson, D. R., Parreira, V. R., Kulkarni, R. R., $\&$ Prescott, J. F. 2006. Live attenuated vaccinebased control of necrotic enteritis of broiler chickens. Veterinary Microbiology, 113(1-2), 25-34.

Tolooe, A., Shojadoost, B., \& Peighambari, S. M. 2011. Molecular detection and characterization of cpb2 gene in Clostridium perfringens isolates from healthy and diseased chickens. Journal of Venomous Animals and Toxins including Tropical Diseases, 17(1), 59-65.

van Immerseel, F., Buck, J., Pasmans, F., Huyghebaert, G., Haesebrouck, F., \& Ducatelle, R. 2004. Clostridium perfringens in poultry: an emerging threat for animal and public health. Avian Pathology, 33(6), 537549.

van Immerseel, F., Rood, J. I., Moore, R. J., \& Titball, R. W. 2009. Rethinking our understanding of the pathogenesis of necrotic enteritis in chickens. Trends in Microbiology, 17(1), 32-36.

Williams, R. B. 2005. Intercurrent coccidiosis and necrotic enteritis of chickens: rational, integrated disease management by maintenance of gut integrity. Avian Pathology, 34(3), 159-180.

Yegani, M., \& Korver, D. R. 2008. Factors affecting intestinal health in poultry. Poultry Science, 87(10), 2052-2063.

Zavala, G. 2007. El clostridium las coccidias y la salmonella desafían a los avicultores que producen pollo de engorde sin antibiticos. Intestinal Health, 3, 16-19.

Recibido: 27 Agosto, 2018.

Aprobado: 7 Septiembre, 2018

Publicado: 14 Septiembre, 2018

Licenciamiento: Este artículo es publicado en la modalidad Acceso Abierto sobre la licencia Creative Commons Atribución 4.0 (CC-BY 4.0), la cual permite uso irrestricto, distribución, reproducción en cualquier medio, desde que el autor y la fuente sean debidamente acreditados. 